\title{
PTEN deficiency is associated with reduced sensitivity to mTOR inhibitor in human bladder cancer through the unhampered feedback loop driving PI3K/Akt activation
}

\author{
E Seront ${ }^{1,2,3}$, A Pinto ${ }^{1}$, C Bouzin ${ }^{1}$, L Bertrand ${ }^{4}$, J-P Machiels ${ }^{2,3}$ and O Feron ${ }^{*, 1}$ \\ ${ }^{1}$ Pole of Pharmacology and Therapeutics, Institut de Recherche Expérimentale et Clinique (IREC), Université catholique de Louvain \\ (UCL), Brussels, Belgium; ${ }^{2}$ Pole of Molecular Imaging, Radiotherapy and Oncology, Institut de Recherche Expérimentale et \\ Clinique (IREC), Université catholique de Louvain (UCL), Brussels, Belgium; ${ }^{3}$ Department of Medical Oncology, Centre du Cancer, \\ Cliniques Universitaires Saint-Luc, Brussels, Belgium and ${ }^{4}$ Pôle de Recherche Cardiovasculaire, Institut de Recherche \\ Expérimentale et Clinique (IREC), Université catholique de Louvain (UCL), Brussels, Belgium
}

Background: Preclinical studies have shown that PTEN loss enhances sensitivity to mammalian target of Rapamycin (mTOR) inhibitors because of facilitated PI3K (phosphatidylinositol-3 kinase)/Akt activation and consecutive stimulation of the mTOR pathway. In patients with advanced transitional cell carcinoma (TCC) treated with the mTOR inhibitor everolimus, PTEN loss was, however, associated with resistance to treatment.

Methods: Transitional cell carcinoma specimens, human bladder cancer cells and derived mouse xenografts were used to evaluate how the PTEN status influences the activity of mTOR inhibitors.

Results: Transitional cell carcinoma patients with a shorter progression-free survival under everolimus exhibited PTEN deficiency and increased Akt activation. Moreover, PTEN-deficient bladder cancer cells were less sensitive to rapamycin than cells expressing wild-type PTEN, and rapamycin strikingly induced Akt activation in the absence of functional PTEN. Inhibition of Akt activation by the PI3K inhibitor wortmannin interrupted this rapamycin-induced feedback loop, thereby enhancing the antiproliferative effects of the mTOR inhibitor both in vitro and in vivo.

Conclusion: Facilitation of Akt activation upon PTEN loss can have a more prominent role in driving the feedback loop in response to mTOR inhibition than in promoting the mTOR pathway. These data support the use of both PI3K and mTOR inhibitors to treat urothelial carcinoma, in particular in the absence of functional PTEN.

The phosphatidylinositol-3 kinase (PI3K)/Akt (Protein Kinase B)/ mammalian target of Rapamycin (mTOR) pathway regulates cell growth, survival and angiogenesis in cancer (Engelman, 2009; Markman et al, 2010; Laplante and Sabatini, 2012). PI3K is a lipid kinase catalysing the formation of phosphatidylinositol-3,4,5 triphosphate (PIP3) from phosphatidylinositol 4,5 bisphosphate (PIP2). PIP3 then recruits phosphoinositide-dependent kinase 1
(PDK1) and Akt to the plasma membrane where Akt is phosphorylated on Thr308 by PDK1, leading to partial activation of Akt (Nicholson and Anderson, 2002). This latter event triggers Ser473 Akt phosphorylation, rendering Akt fully active. Activation of mTOR by Akt stimulates protein synthesis through the actions of the ribosomal protein S6 kinase (S6K) and the translational initiation factor eIF-4E-binding protein 1 (4EBP1) (Guertin and 
Sabatini, 2007; Meric-Bernstam and Gonzalez-Angulo, 2009). A negative regulatory feedback loop exists between mTOR and PI3K, which is mediated by S6K-dependent phosphorylation of Insulin Receptor Substrate-1 (IRS-1) - the substrate of the tyrosine kinase receptor coupled to PI3K (Sun et al, 2005; O'Reilly et al, 2006). Inhibition of mTOR can thus lead to Akt activation, which can phosphorylate numerous substrates, thereby promoting cell proliferation and survival (Chandarlapaty et al, 2011).

The phosphatase and tensin homologue deleted on chromosome 10 (PTEN) acts as a negative regulator of PI3K by dephosphorylating PIP3, resulting in a decreased activation of its downstream targets including Akt (Maehama and Dixon, 1998; Stambolic et al, 1998). It is well demonstrated that the PI3K/Akt/ mTOR pathway is constitutively activated in a variety of human cancer mostly because of the loss of PTEN (Hollander et al, 2011). In particular, PTEN loss of heterozygosity or PTEN decreased expression has been observed in a large number of invasive urothelial carcinoma (UC) (Aveyard et al, 1999; Tsuruta et al, 2006; Platt et al, 2009; Qian et al, 2009). Several preclinical models have further shown that PTEN-deficient tumours present an enhanced sensitivity to mTOR inhibitors because of a sustained activation of PI3K/Akt signaling (Neshat et al, 2001; Podsypanina et al, 2001; Shi et al, 2002; Steelman et al, 2008). These experimental observations have encouraged clinical trials aiming to evaluate mTOR inhibitors in different cancer types, including bladder cancer, mostly represented by transitional carcinoma cell (TCC). Recently, we reported the results of a phase II trial documenting the clinical activity of the mTOR inhibitor everolimus in patients with advanced bladder TCC after failure of platinum-based chemotherapy (Seront et al, 2012). Interestingly, using archival tumour samples of these patients, we found that PTEN loss was paradoxically only observed in patients resistant to everolimus.

In the current study, we have therefore examined the link between PTEN expression and the status of the mTOR pathway. A negative correlation between PTEN expression and Akt phosphorylation (Ser 473) in human TCC specimens led us to explore whether this pathway could account for the resistance (instead of the sensitivity) to the mTOR inhibitor rapamycin in mouse models of bladder cancer. We found that PTEN-deficient bladder tumour cells were indeed more resistant to rapamycin than PTEN-positive cells because of their inability to abrogate the activation of the prosurvival Akt signaling cascade induced by mTOR inhibition itself. We also demonstrated that pharmacological inhibition of PI3K could enhance the therapeutic effects of rapamycin, particularly in PTEN-deficient bladder tumours.

\section{MATERIALS AND METHODS}

Cancer patient tissue samples. Formalin-fixed paraffinembedded (FFPE) samples were archival tumour tissues retrieved from patients enrolled in a phase II trial that evaluated efficacy of everolimus in advanced TCC (Seront et al, 2012). Disease control rate at 8 weeks, including complete response, partial response and stable disease, was the primary end point of this trial (see Seront et al (2012) for details). Tissue samples harbouring PIK3CA mutations were excluded for this study, leaving 15 tissue samples for immunohistochemical analyses, 5 from patients with controlled disease and 10 from patients with non-controlled disease upon everolimus.

Tumour cells and in vitro treatments. Human bladder cell lines UM-UC-3, UM-UC-9 and UM-UC-14 were acquired from ECACC, in which they are regularly authenticated. Cells were stored according to the supplier's instructions, used within 6 months after resuscitation of frozen aliquots and cultured as recommended by ECACC. Cell proliferation was determined in 96-well plates using crystal violet after treatment or not with rapamycin or wortmannin (LC Laboratory, Woburn, MA, USA). In some experiments, UM-UC-3 cells were transfected with a plasmid encoding wild-type PTEN (Addgene, Cambridge, MA, USA) (or the corresponding empty vector as control) (Ramaswamy et al, 1999) using the X-tremeGENE 9 reagent (Roche, Penzberg, Germany).

Mouse models and in vivo treatments. Eight weeks old female NMRI nude mice (Elevage Janvier, LeGenest-St-Isle, France) were injected subcutaneously with $3 \times 10^{6}$ tumour cells in the right dorsal flank. Tumours were allowed to reach a minimal diameter of $5 \mathrm{~mm}$, and the animals (five to six mice per group and per experiment) were then injected every 2 days with either DMSO (vehicle), rapamycin $\left(0.75 \mathrm{mg} \mathrm{kg}^{-1}\right.$ per day) and/or wortmannin ( $1 \mathrm{mg} \mathrm{kg}^{-1}$ per day). Tumour sizes were tracked with an electronic caliper, and at the killing tumour samples were frozen in liquid nitrogen. These procedures were approved by the local authorities according to the national animal care regulations.

Immunoblotting and immunostaining. For immunoblotting, cell extracts were separated by SDS-PAGE and transferred onto PVDF membranes before incubation with primary antibodies; gel loading was normalised with a $\beta$-actin antibody (Sigma, St Louis, MO, USA). Antibodies against total or phosphorylated proteins were all from Cell Signaling (Danvers, MA, USA) except the anti-phosphoThr308 Akt antibody, which was from Abcam (Cambridge, UK). For immunocytochemistry, cells were seeded in eight-chamber slides (Labtek, Roskilde, Denmark), treated and fixed before staining; secondary antibodies coupled to Alexa 488 were used and the nuclei were counterstained with DAPI. Immunohistochemistry was performed on FFPE tissue sections, as previously described (Seront et al, 2012). Staining was evaluated by two independent observers to establish corresponding histoscores ( $H$-score), using the following formula: percentage of weakly stained $\times 1+$ percentage of moderately stained $\times 2+$ percentage of strongly stained $\times 3$. In correlation analyses, a cutoff value based on the median tumour $H$-score was used so that protein expression was considered as low when <median $H$-score and high when $\geqslant$ median $H$-score.

Statistical analysis. Association between PTEN expression and everolimus sensitivity was calculated using the Fisher's test. Correlation between the extents of protein expression/phosphorylation was calculated using Spearman's analysis. Progression-free survival (PFS) related to PTEN expression was analysed using the Kaplan-Meier method, and curves were compared using a log-rank test. In all the other settings, results are presented as mean \pm s.e.m. and statistical analyses were carried out using two-way ANOVA tests (followed by a Bonferroni post-test).

\section{RESULTS}

Deficient PTEN expression is associated with non-controlled disease and Akt phosphorylation in advanced TCC patients treated with everolimus. We have previously reported a possible link between PTEN loss and everolimus resistance in patients with advanced bladder TCC (Seront et al, 2012). In the current study, using archival tumours (not displaying PI3KCA mutations), we found that patients with no or low tumour expression of PTEN (Supplementary Figure 1) had a shorter PFS than patients with PTEN expression (median survival 61 vs 119 days, respectively, exploratory log-rank $P=0.002$ ) (Figure 1 ). We then examined the association between PTEN expression, the phosphorylations of status of Akt, mTOR and S6RP (used as markers of mTOR/S6K activation), and the disease control under everolimus treatment. 
The expression of PTEN was significantly associated $(P=0.02)$ with controlled disease (Table 1). Among the above signaling proteins, Akt phosphorylation (Ser473) was positively correlated with $\mathrm{mTOR} / \mathrm{S} 6 \mathrm{RP}$ phosphorylation $(P=0.02)$ and negatively with the expression of PTEN $(P=0.054)$ (Table 2$)$. Other posphorylated sites/proteins including phospho-Thr308 Akt, phospho-Ser2448 mTOR and phospho-Ser235/236 S6RP failed to display association with the PTEN status (Table 2).

Rapamycin induces Akt activation in resistant PTEN-deficient bladder cancer cells. To better understand the influence of PTEN expression on the efficacy of mTOR inhibitors, we used three human bladder cancer cell lines with different PTEN status.

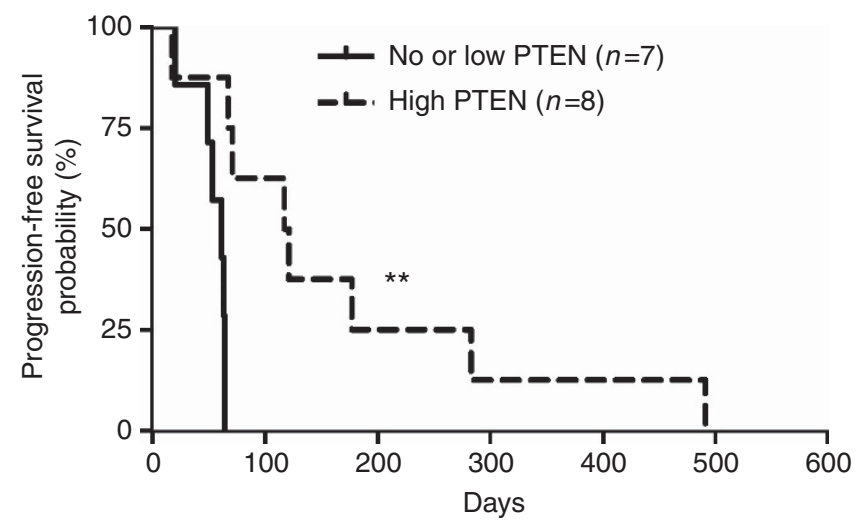

Figure 1. Survival analyses of TCC using PTEN histopathology as an prognostic indicator. Comparison of Kaplan-Meier curves of PFS according to PTEN expression was performed using exploratory log-rank test; ${ }^{\star \star} P<0.01$.

Table 1. Relationship between disease control in everolimus-treated TCC patients vs PTEN expression or the status of mTOR/Akt/S6RP phosphorylations

\begin{tabular}{|l|c|}
\hline & Correlation with disease control \\
\hline PTEN & $P=0.02$ \\
\hline phospho-Ser473 Akt & $P=0.60$ \\
\hline phospho-Thr308 Akt & $P=1.00$ \\
\hline phospho-Ser2448 mTOR & $P=0.95$ \\
\hline Phospho-Ser235/236 S6RP & $P=0.95$ \\
\hline $\begin{array}{l}\text { Abbreviations: } m \text { TOR }=\text { mammalian target of Rapamycin; TCC = transitional cell carcinoma. } \\
\text { The relative extent of protein expression/phosphorylation was compared using calculated } \\
\text { H-scores. }\end{array}$ \\
\hline
\end{tabular}

UM-UC-3 cells show homozygous deletion of PTEN, UM-UC-9 cells have reduced PTEN expression and UM-UC-14 cells express wild-type PTEN. These three cell lines were treated with increasing doses of the mTOR inhibitor rapamycin for $72 \mathrm{~h}$ (Figure 2A). Whereas the UM-UC-14 cells were highly sensitive to mTOR inhibition, with a 50\% decrease in cell proliferation in the presence of 1 nM rapamycin, UM-UC-9 and UM-UC-3 showed a 15\% and $5 \%$ reduction in cell density following exposure to $100 \mathrm{~nm}$ rapamycin ( $v s$ untreated cells), respectively. Immunoblotting experiments further documented that S6RP phosphorylation on serines 235/236 was similarly fully inhibited in either UM-UC-3 or UM-UC-14 cells (Figure 2B), confirming the efficient inhibition of mTOR activity with rapamycin independently of the PTEN status. Contrasting effects on Akt activation were however observed in response to rapamycin. Whereas a net increase in Ser473 Akt phosphorylation was observed in rapamycin-treated UM-UC-3 cells, no change was observed in UM-UC-14 cells (Figure 2B). Immunocytochemistry confirmed that rapamycin induced a dramatic increase in Ser473 Akt phosphorylation in PTENdeficient UM-UC-3 cells but failed to do so in PTEN-expressing UM-UC-14 (Figure 2C).

Resistance to rapamycin in PTEN-deficient cancer cells is relieved by the PI3K inhibitor wortmannin. To investigate the possible role of Akt activation in the development of rapamycin resistance, we used the PI3K inhibitor wortmannin. We found that wortmannin alone reduced the proliferation of UM-UC-3 and UM-UC- 14 cells by $12 \%$ and $48 \%$, respectively (Figure $3 \mathrm{~A}$ ). In UM-UC-14 cells, the association of wortmannin with rapamycin resulted in a slightly higher toxicity ( $v s$ rapamycin alone) (Figure 3A). More interestingly, in PTEN-deficient UM-UC-3 cells, addition of wortmannin synergistically $(P<0.01)$ enhanced the toxicity of rapamycin, reducing cell proliferation by $40 \%$ when compared with rapamycin alone (Figure 3A). Immunoblotting experiments showed that wortmannin completely abrogated the increase in Ser473 Akt phosphorylation in rapamycin-treated UMUC-3 cells (Figures $3 \mathrm{~B}$ and C, left panel). Detection of the phosphorylated form (Ser 9) of GSK3, a bona fide effector of Akt activation, further confirmed that in PTEN-deficient UM-UC-3 cells, rapamycin treatment significantly stimulated the pro-survival Akt/GSK3 pathway in a wortmannin-sensitive manner, whereas in PTEN-expressing UM-UC-14 cells, GSK3 phosphorylation was prevented by rapamycin (Figure 3B). Interestingly, wortmannin alone significantly reduced the extent of Ser235/236 S6RP phosphorylation in both UM-UC-3 ( $-35 \%)$ and UM-UC-14 cells (-95\%) (Figures 3B and D).

Wortmannin sensitises PTEN-deficient UM-UC-3 tumours to mTOR inhibition in vivo. We then explored the effects of rapamycin and wortmannin on the in vivo growth of bladder tumours. Mice were injected s.c. with either UM-UC-3 and

Table 2. Relationship between the expression of PTEN and the phosphorylation status of Akt, mTOR and S6RP in human TCC specimens

\begin{tabular}{|c|c|c|c|c|}
\hline & PTEN & phospho-Ser473 Akt & phospho-Thr308 Akt & phospho-Ser2448 mTOR \\
\hline phospho-Ser473 Akt & $\begin{array}{l}R=-0.5 \\
P=0.054\end{array}$ & & & \\
\hline phospho-Thr308 Akt & $\begin{array}{c}R=-0.24 \\
P=0.31\end{array}$ & $\begin{array}{l}R=0.38 \\
P=0.15\end{array}$ & & \\
\hline phospho-Ser2448 mTOR & $\begin{array}{c}R=-0.07 \\
P=0.79\end{array}$ & $\begin{array}{l}R=0.58 \\
P=0.02\end{array}$ & $\begin{array}{l}R=0.09 \\
P=0.73\end{array}$ & \\
\hline phospho-Ser235/236 S6RP & $\begin{array}{c}R=-0.07 \\
P=0.79\end{array}$ & $\begin{array}{l}R=0.59 \\
P=0.02\end{array}$ & $\begin{array}{l}R=0.09 \\
P=0.73\end{array}$ & $\begin{array}{c}R=0.95 \\
P<0.001\end{array}$ \\
\hline
\end{tabular}


A

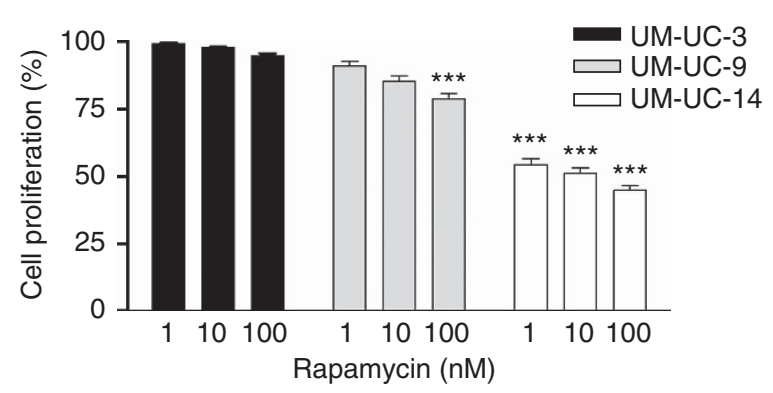

B

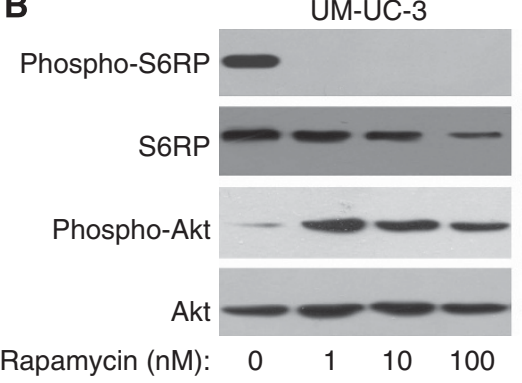

C
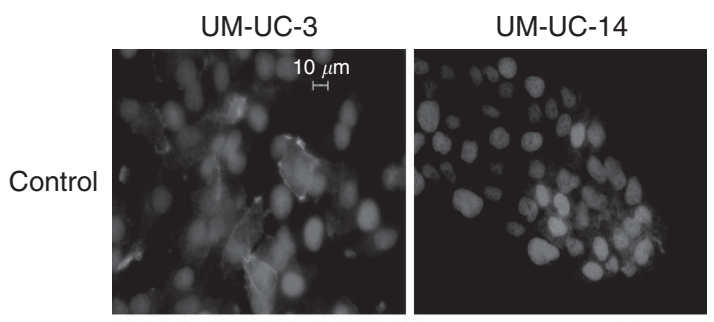

Rapamycin $1 \mathrm{nM}$
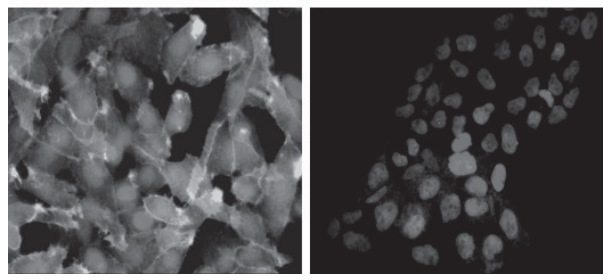

Figure 2. Resistance to rapamycin in PTEN-deficient bladder cancer cells is associated with increased Akt phosphorylation. (A) Extent of UM-UC-3, UM-UC-9 and UM-UC-14 cell proliferation (expressed as $\%$ of control) after $72 \mathrm{~h}$ treatment with the indicated doses of rapamycin ( ${ }^{\star \star \star} P<0.001, n=3$ independent experiments performed in triplicate). (B) Representative immunoblots obtained from UM-UC-3 and UM-UC-14 cells treated for $48 \mathrm{~h}$ with increasing doses of rapamycin; this experiment was repeated three times with similar results.

(C) Representative immunofluorescence detection (green) of phosphoSer473 Akt in UM-UC-3 and UM-UC-14 cells exposed or not to $10 \mathrm{~nm}$ rapamycin for $48 \mathrm{~h}$; nuclei were counterstained (blue) with DAPI. This experiment was repeated three times with similar results. The full colour version of this figure is available at British Journal of Cancer online.

UM-UC-14, and when the corresponding tumours reached a diameter of $5 \mathrm{~mm}$, they were treated with rapamycin, wortmannin or both. Consistently with the association between PTEN loss and disease aggressiveness observed in patients, UM-UC-3 xenografts presented a more rapid growth than UM-UC-14 xenografts (Figure 4A). In UM-UC-3 xenografts, wortmannin alone had no significant effect on tumour growth but deeply increased the antitumor effects of rapamycin when both drugs were administered concomitantly (Figure 4A, left panel). In UM-UC-14, rapamycin and wortmannin, used as monotherapy, inhibited tumour growth; combination of both, however, had no further impact (Figure 4A, right panel).
Immunohistochemical analysis of corresponding tumour sections showed that (i) rapamycin increased Akt phosphorylation in UM-UC-3 cells but not in PTEN-expressing UM-UC-14 cells (Figure $4 \mathrm{~B}$ and Supplementary Figure 2A), whereas it completely inhibited S6RP phosphorylation in both cell types (Figure 4C and Supplementary Figure 2B), (ii) wortmannin associated with rapamycin completely blocked the phosphorylation of both Akt and S6RP in both cell phenotypes (Figure 4B and Supplementary Figures $2 \mathrm{~A}$ and $\mathrm{B}$ ). Of note, wortmanin used alone barely influenced the extent of phospho-S6RP in UM-UC-3 cells but completely inhibited it in UM-UC-14 cells (Figure 4C and Supplementary Figure 2B).

PTEN re-expression in PTEN-negative UM-UC-3 restores the sensitivity to rapamycin. To definitively prove the role of PTEN loss in the resistance to mTOR inhibitor observed both in vitro and in vivo, we transfected PTEN-deficient UM-UC-3 cells with a PTEN-expressing plasmid (Figure 5A). We found that PTEN re-expression led to a significant decrease in cell proliferation in response to rapamycin $(P<0.01)$ (Figure $5 \mathrm{~B})$. Immunoblotting experiments further showed that the expression of PTEN in UM-UC-3 cells prevented the phosphorylation of Akt in response to rapamycin treatment (Figures $5 \mathrm{C}$ and D for quantification).

\section{DISCUSSION}

The current study was initiated following a phase II clinical trial, which led us to document that PTEN loss was unexpectedly associated with resistance to the mTOR inhibitor everolimus in patients with advanced bladder cancer (Seront et al, 2012). These results were indeed in contrast with the anticipated stimulation of the mTOR pathway by the upstream PI3K/Akt activation unhampered by the phosphatase PTEN. In the current study, the identification of a correlation between PTEN loss and Akt phosphorylation in TCC patients led us to postulate that instead of stimulating mTOR and thereby sensitising tumours to inhibitors of this pathway, Akt activation had a pro-survival role opposing the antitumour effects of mTOR inhibition. Using bladder cancer cell lines with distinct PTEN status, we actually found that PTEN loss can facilitate a negative feedback loop leading to PI3K/Akt activation in response to rapamycin treatment. Furthermore, the use of the PI3K inhibitor wortmannin led us to document both in vitro and in vivo that the prevention of rapamycin-induced Akt activation sensitised PTEN-deficient bladder cancer cells to mTOR inhibition. Moreover, PTEN re-expression in these PTEN-deficient cells prevented Akt activation and restored the sensitivity to rapamycin. In bladder tumour cells expressing wild-type PTEN, we did not observe rapamycin-induced activation of Akt, confirming the inhibitory role of PTEN on the PI3K/Akt signaling cascade and in agreement with the lack of Akt activation in PTEN-positive TCC patients responding to everolimus.

Interestingly, the basal activity of Akt in PTEN-expressing bladder cancer cells was associated with a higher phosphorylation level of GSK3 than that in the PTEN-deficient cells (Figure 3B). Moreover, in cells with functional PTEN, rapamycin completely abrogated Akt phosphorylation and dramatically decreased GSK3 phosphorylation (Figure 3B). These observations indicate that at least part of Akt activity is under the control of mTOR in PTENexpressing cells. This observation warrants further investigation to determine whether this involves the mTORC2 complex known to support Akt activation (Guertin and Sabatini, 2007; MericBernstam and Gonzalez-Angulo, 2009; Vilar et al, 2011). Interestingly, wortmannin used alone could actually reduce the extent of phospho-S6RP in both PTEN phenotypes in vitro (Figure $3 \mathrm{~B}$ ) and in vivo (Figures $4 \mathrm{D}$ and $\mathrm{E}$ ). Although the capacity of wortmannin 
A

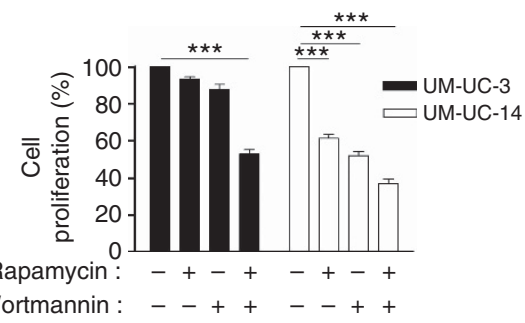

B

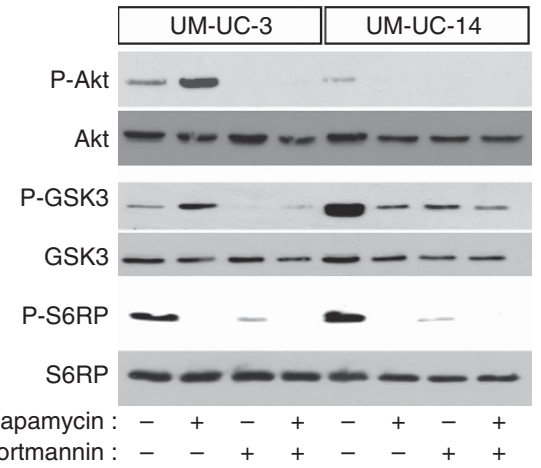

C

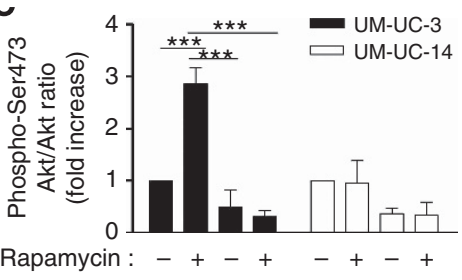

Wortmannin: --++--++

D

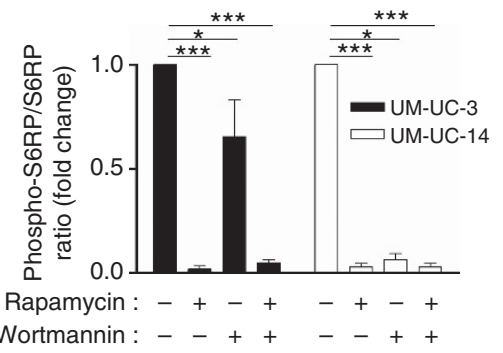

Figure 3. Wortmannin sensitizes PTEN-deficient bladder cancer cells to rapamycin. (A) Extent of UM-UC-3 and UM-UC-14 cell proliferation (expressed as \% of control) after 72 -h treatment with $1 \mathrm{~nm}$ rapamycin, $5 \mu \mathrm{m}$ wortmannin or both ( ${ }^{* \star} P<0.001, n=3$ independent experiments performed in triplicate). (B) Representative immunoblots obtained from UM-UC-3 and UM-UC-14 cells treated for $48 \mathrm{~h}$ as described above. Quantification of (C) phospho-Akt/Akt and (D) phospho-S6RP/S6RP ratios determined from different immunoblots and expressed as \% of control $\left({ }^{\star} P<0.05,{ }^{* \star *} P<0.001, n=3\right)$.

A

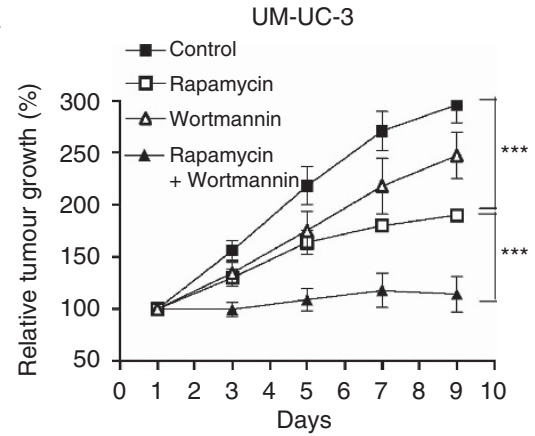

B

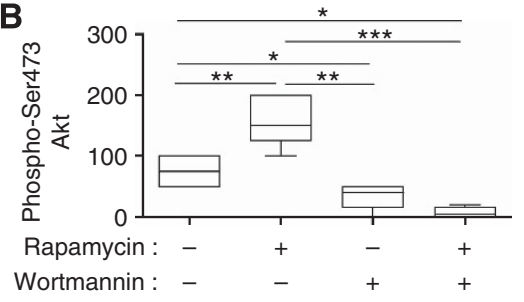

C

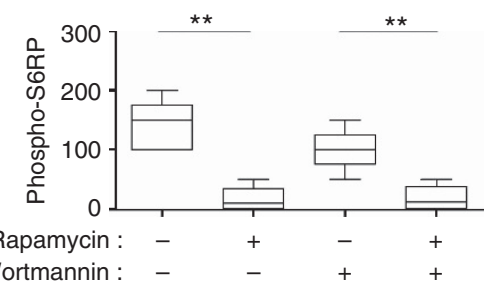

UM-UC-14
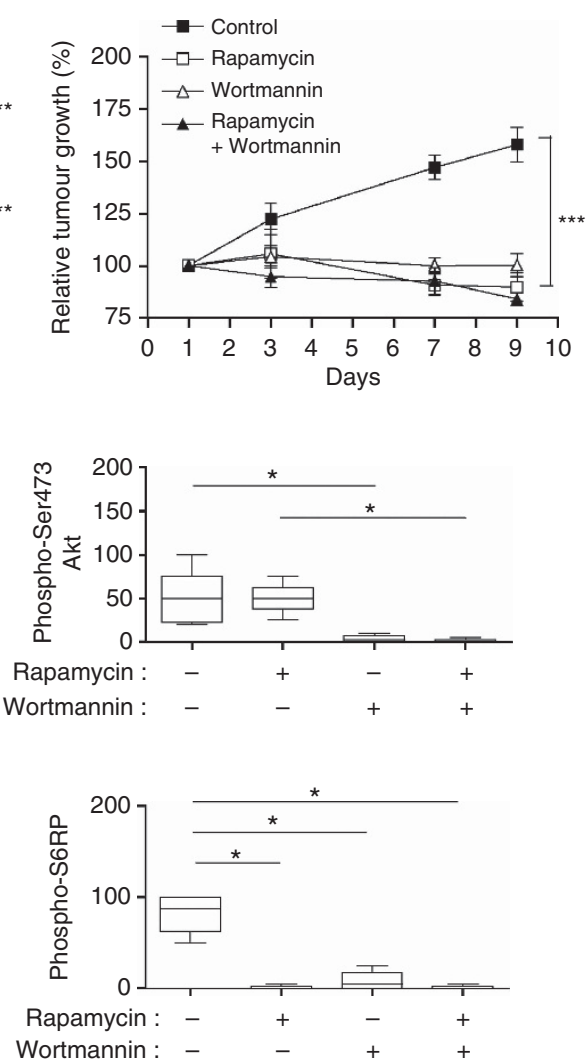

Figure 4. Wortmannin prevents rapamycin-induced Akt phosphorylations in PTEN-deficient tumours and sensitizes them to the antiproliferative effects of mTOR inhibition. Mice bearing UM-UC-3 (left) and UM-UC-14 (right) tumours were treated with rapamycin $\left(0.5 \mathrm{mg} \mathrm{kg}{ }^{-1}\right.$ per day), wortmannin $\left(0.75 \mathrm{mg} \mathrm{kg}^{-1}\right.$ per day) or both (through intraperitoneal administration each 2 days, five mice per group). (A) Evolution of tumour growth in the different conditions $(* \star \star P<0.001)$. H-score quantification of immunostaining for (B) phospho-Ser473 Akt and (C) phospho-Ser235/ 236 S6RP in sections of corresponding tumors ( ${ }^{\star} P<0.05,{ }^{\star \star} P<0.01,{ }^{\star \star \star} P<0.001, n=5$ mice per condition). 
A

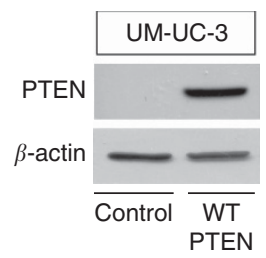

C

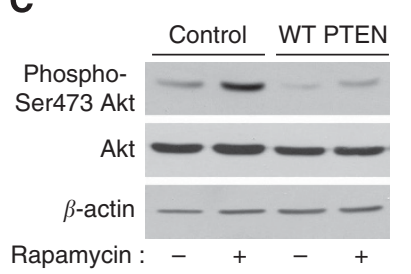

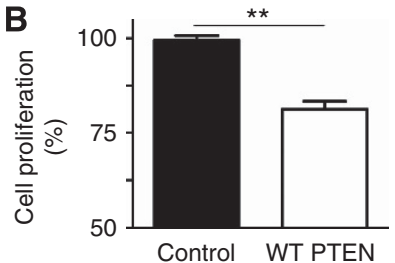

D

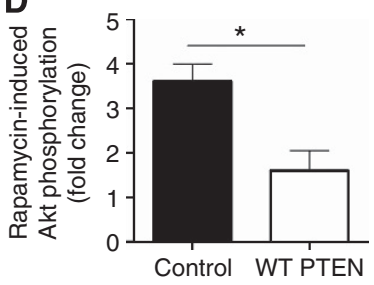

Figure 5. Re-expression of PTEN in PTEN-deficient bladder tumour cells prevents rapamycin-induced Akt phosphorylations and restores sensitivity to mTOR inhibition. PTEN-deficient UM-UC-3 cells were transfected with wild-type PTEN-encoding plasmid or corresponding empty vector. (A) Representative PTEN immunoblot. (B) Extent of cell proliferation (expressed as \% of control) after 48-h treatment with $1 \mathrm{nM}$ rapamycin ( ${ }^{*} P<0.01, n=3$ independent experiments performed in triplicate). (C) Representative phospho-Akt and total Akt immunoblots after same treatment; $\beta$-actin was used as loading control.

(D) Quantification of rapamycin-induced increase in phospho-Akt/Akt ratio determined from different immunoblots and expressed as fold-change (vs corresponding untreated cells); ${ }^{\star} P<0.05, n=3$ independent experiments performed in triplicate.

to also interfere with mTOR activity (Brunn et al, 1996; Feldman and Shokat, 2010) could partly account for these effects, the action of the PI3K inhibitor as monotherapy was more pronounced in the presence of functional PTEN (Figures $3 \mathrm{~B}$ and $4 \mathrm{C}$ ). Altogether, these data indicate that inhibition of Akt can not only sensitise PTEN-deficient tumour cells to the effects of mTOR inhibition but can also cooperate with mTOR inhibitors to exert antitumour effects in wild-type PTEN-expressing cancer types.

This exploratory study therefore positions the tumour PTEN status as an additional layer of regulation in the previously reported Akt-driven feedback loop associated with resistance to mTOR inhibitors (Sun et al, 2005; O'Reilly et al, 2006; Yohn et al, 2011). This suggests that clinically the loss of PTEN should not automatically be associated with an increased sensitivity to mTOR inhibitors because of an expected increased activation of the PI3K/ Akt pathway. Although this association may be true and was reported in different preclinical studies (Neshat et al, 2001; Podsypanina et al, 2001; Shi et al, 2002; Steelman et al, 2008), we have documented here an opposite relationship in TCC patients and in mice bearing bladder tumours. In these settings, PTEN loss may actually decrease the antitumoral efficacy of mTOR inhibitors. Limitations to our work should however be emphasised, considering the limited number of enrolled TCC patients and the use of human bladder cancer cell lines exhibiting distinct PTEN status but being non-isogenic and thus with other possible alterations in the PI3K/Akt/mTOR axis. Still, in support to our observations, it is noteworthy that Cloughesy et al (2008) previously reported that 1-week administration of rapamycin before the surgery of glioblastoma lacking PTEN expression induced Akt activation in the subset of patients with the shorter time-to-progression. More generally, our study could also explain the poor predictive value of PTEN loss to anticipate the efficacy of mTOR inhibitors in clinical trials (Figlin et al, 2009; Javle et al, 2010; Oza et al, 2011; Fleming et al, 2012; Tredan et al, 2012). Recently, using 31 breast cancer cell lines, Weigelt et al (2011) could exclude that cells harbouring

PTEN loss were more sensitive to mTOR inhibitors. More definitive validation of our work, however, would require to prospectively stratify cancer patients according to their tumour PTEN status in future clinical trials evaluating rapalogs as anticancer agents. Finally, our results give more credential to the association of mTOR and PI3K inhibitors or the use of drugs combining both activities (Markman et al, 2010), as such strategy should be beneficial both for tumours expressing or not wild-type PTEN.

\section{ACKNOWLEDGEMENTS}

This work was supported by grants from the Fonds de la Recherche Scientifique FRS-FNRS, the Télévie, the Foundation against cancer, the J Maisin Foundation, an Action de Recherche Concertée (ARC 09/14-020) and the interuniversity attraction pole (IUAP programme P7.03). LB is a Research Associate of the Fonds National de la Recherche Scientifique.

\section{CONFLICT OF INTEREST}

The authors declare no conflict of interest.

\section{REFERENCES}

Aveyard JS, Skilleter A, Habuchi T, Knowles MA (1999) Somatic mutation of PTEN in bladder carcinoma. Br J Cancer 80: 904-908.

Brunn GJ, Williams J, Sabers C, Wiederrecht G, Lawrence Jr JC, Abraham RT (1996) Direct inhibition of the signaling functions of the mammalian target of rapamycin by the phosphoinositide 3-kinase inhibitors, wortmannin and LY294002. EMBO J 15: 5256-5267.

Chandarlapaty S, Sawai A, Scaltriti M, Rodrik-Outmezguine V, GrbovicHuezo O, Serra V, Majumder PK, Baselga J, Rosen N (2011) AKT inhibition relieves feedback suppression of receptor tyrosine kinase expression and activity. Cancer Cell 19: 58-71.

Cloughesy TF, Yoshimoto K, Nghiemphu P, Brown K, Dang J, Zhu S, Hsueh T, Chen Y, Wang W, Youngkin D, Liau L, Martin N, Becker D, Bergsneider M, Lai A, Green R, Oglesby T, Koleto M, Trent J, Horvath S, Mischel PS, Mellinghoff IK, Sawyers CL (2008) Antitumor activity of rapamycin in a phase I trial for patients with recurrent PTEN-deficient glioblastoma. PLoS Med 5: e8.

Engelman JA (2009) Targeting PI3K signalling in cancer: opportunities, challenges and limitations. Nat Rev Cancer 9: 550-562.

Feldman ME, Shokat KM (2010) New inhibitors of the PI3K-Akt-mTOR pathway: insights into mTOR signaling from a new generation of Tor Kinase Domain Inhibitors (TORKinibs). Curr Top Microbiol Immunol 347: 241-262.

Figlin RA, De SP, McDermott D, Dutcher JP, Berkenblit A, Thiele A, Krygowski M, Strahs A, Feingold J, Boni J, Hudes G (2009) Analysis of PTEN and HIF-1alpha and correlation with efficacy in patients with advanced renal cell carcinoma treated with temsirolimus versus interferon-alpha. Cancer 115: 3651-3660.

Fleming GF, Ma CX, Huo D, Sattar H, Tretiakova M, Lin L, Hahn OM, Olopade FO, Nanda R, Hoffman PC, Naughton MJ, Pluard T, Conzen SD, Ellis MJ (2012) Phase II trial of temsirolimus in patients with metastatic breast cancer. Breast Cancer Res Treat 136: 355-363.

Guertin DA, Sabatini DM (2007) Defining the role of mTOR in cancer. Cancer Cell 12: 9-22.

Hollander MC, Blumenthal GM, Dennis PA (2011) PTEN loss in the continuum of common cancers, rare syndromes and mouse models. Nat Rev Cancer 11: 289-301.

Javle MM, Shroff RT, Xiong H, Varadhachary GA, Fogelman D, Reddy SA, Davis D, Zhang Y, Wolff RA, Abbruzzese JL (2010) Inhibition of the mammalian target of rapamycin (mTOR) in advanced pancreatic cancer: results of two phase II studies. BMC Cancer 10: 368 .

Laplante M, Sabatini DM (2012) mTOR signaling in growth control and disease. Cell 149: 274-293. 
Maehama T, Dixon JE (1998) The tumour suppressor, PTEN/MMAC1, dephosphorylates the lipid second messenger, phosphatidylinositol 3,4,5-trisphosphate. J Biol Chem 273: 13375-13378.

Markman B, Dienstmann R, Tabernero J (2010) Targeting the PI3K/Akt/ mTOR pathway-beyond rapalogs. Oncotarget 1: 530-543.

Meric-Bernstam F, Gonzalez-Angulo AM (2009) Targeting the mTOR signaling network for cancer therapy. J Clin Oncol 27: 2278-2287.

Neshat MS, Mellinghoff IK, Tran C, Stiles B, Thomas G, Petersen R, Frost P, Gibbons JJ, Wu H, Sawyers CL (2001) Enhanced sensitivity of PTENdeficient tumours to inhibition of FRAP/mTOR. Proc Natl Acad Sci USA 98: 10314-10319.

Nicholson KM, Anderson NG (2002) The protein kinase B/Akt signalling pathway in human malignancy. Cell Signal 14: 381-395.

O’Reilly KE, Rojo F, She QB, Solit D, Mills GB, Smith D, Lane H, Hofmann F, Hicklin DJ, Ludwig DL, Baselga J, Rosen N (2006) mTOR inhibition induces upstream receptor tyrosine kinase signaling and activates Akt. Cancer Res 66: 1500-1508.

Oza AM, Elit L, Tsao MS, Kamel-Reid S, Biagi J, Provencher DM, Gotlieb WH, Hoskins PJ, Ghatage P, Tonkin KS, Mackay HJ, Mazurka J, Sederias J, Ivy P, Dancey JE, Eisenhauer EA (2011) Phase II study of temsirolimus in women with recurrent or metastatic endometrial cancer: a trial of the NCIC Clinical Trials Group. J Clin Oncol 29: 3278-3285.

Platt FM, Hurst CD, Taylor CF, Gregory WM, Harnden P, Knowles MA (2009) Spectrum of phosphatidylinositol 3-kinase pathway gene alterations in bladder cancer. Clin Cancer Res 15: 6008-6017.

Podsypanina K, Lee RT, Politis C, Hennessy I, Crane A, Puc J, Neshat M, Wang H, Yang L, Gibbons J, Frost P, Dreisbach V, Blenis J, Gaciong Z, Fisher P, Sawyers C, Hedrick-Ellenson L, Parsons R (2001) An inhibitor of mTOR reduces neoplasia and normalizes p70/S6 kinase activity in Pten + / - mice. Proc Natl Acad Sci USA 98: 10320-10325.

Qian CN, Furge KA, Knol J, Huang D, Chen J, Dykema KJ, Kort EJ, Massie A, Khoo SK, Vanden Beldt K, Resau JH, Anema J, Kahnoski RJ, Morreau H, Camparo P, Comperat E, Sibony M, Denoux Y, Molinie V, Vieillefond A, Eng C, Williams BO, Teh BT (2009) Activation of the PI3K/AKT pathway induces urothelial carcinoma of the renal pelvis: identification in human tumours and confirmation in animal models. Cancer Res 69: 8256-8264.

Ramaswamy S, Nakamura N, Vazquez F, Batt DB, Perera S, Roberts TM, Sellers WR (1999) Regulation of G1 progression by the PTEN tumour suppressor protein is linked to inhibition of the phosphatidylinositol 3-kinase/Akt pathway. Proc Natl Acad Sci USA 96: 2110-2115.

Seront E, Rottey S, Sautois B, Kerger J, D’Hondt LA, Verschaeve V, Canon JL, Dopchie C, Vandenbulcke JM, Whenham N, Goeminne JC, Clausse M, Verhoeven D, Glorieux P, Branders S, Dupont P, Schoonjans J, Feron O, Machiels JP (2012) Phase II study of everolimus in patients with locally advanced or metastatic transitional cell carcinoma of the urothelial tract: clinical activity, molecular response, and biomarkers. Ann Oncol 23: 2663-2670.

Shi Y, Gera J, Hu L, Hsu JH, Bookstein R, Li W, Lichtenstein A (2002) Enhanced sensitivity of multiple myeloma cells containing PTEN mutations to CCI-779. Cancer Res 62: 5027-5034.

Stambolic V, Suzuki A, de la Pompa JL, Brothers GM, Mirtsos C, Sasaki T, Ruland J, Penninger JM, Siderovski DP, Mak TW (1998) Negative regulation of $\mathrm{PKB} / \mathrm{Akt}$-dependent cell survival by the tumour suppressor PTEN. Cell 95: 29-39.

Steelman LS, Navolanic PM, Sokolosky ML, Taylor JR, Lehmann BD, Chappell WH, Abrams SL, Wong EW, Stadelman KM, Terrian DM, Leslie NR, Martelli AM, Stivala F, Libra M, Franklin RA, McCubrey JA (2008) Suppression of PTEN function increases breast cancer chemotherapeutic drug resistance while conferring sensitivity to mTOR inhibitors. Oncogene 27: 4086-4095.

Sun SY, Rosenberg LM, Wang X, Zhou Z, Yue P, Fu H, Khuri FR (2005) Activation of Akt and eIF4E survival pathways by rapamycin-mediated mammalian target of rapamycin inhibition. Cancer Res 65: 7052-7058.

Tredan O, Treilleux I, Wang Q, Gane N, Pissaloux D, Bonnin N, Petit T, Cretin J, Bonichon-Lamichhane N, Priou F, Lavau-Denes S, Mari V, Freyer G, Lebrun D, Alexandre J, Ray-Coquard I (2012) Predicting everolimus treatment efficacy in patients with advanced endometrial carcinoma: a GINECO group study. Target Oncol; e-pub ahead of print 13 December 2012; doi:10.1007/s11523-012-0242-9.

Tsuruta H, Kishimoto H, Sasaki T, Horie Y, Natsui M, Shibata Y, Hamada K, Yajima N, Kawahara K, Sasaki M, Tsuchiya N, Enomoto K, Mak TW, Nakano T, Habuchi T, Suzuki A (2006) Hyperplasia and carcinomas in Pten-deficient mice and reduced PTEN protein in human bladder cancer patients. Cancer Res 66: 8389-8396.

Vilar E, Perez-Garcia J, Tabernero J (2011) Pushing the envelope in the mTOR pathway: the second generation of inhibitors. Mol Cancer Ther 10: $395-403$.

Weigelt B, Warne PH, Downward J (2011) PIK3CA mutation, but not PTEN loss of function, determines the sensitivity of breast cancer cells to mTOR inhibitory drugs. Oncogene 30: 3222-3233.

Yohn NL, Bingaman CN, DuMont AL, Yoo LI (2011) Phosphatidylinositol 3'-kinase, mTOR, and glycogen synthase kinase-3beta mediated regulation of p21 in human urothelial carcinoma cells. BMC Urol 11: 19.

This work is published under the standard license to publish agreement. After 12 months the work will become freely available and the license terms will switch to a Creative Commons AttributionNonCommercial-Share Alike 3.0 Unported License.

Supplementary Information accompanies this paper on British Journal of Cancer website (http://www.nature.com/bjc) 